\section{Chronic cerebrospinal venous insufficiency, ten years after. New headlights on a venous disease that enriched the vascular world}

\section{Pietro M. Bavera}

Vascular Surgeon and Diagnostician for Solferino Vascular Lab, Milano; Member of the Italian Society for Angiology and Vascular Medicine (SIAPAV); Member of the Italian Society for Vascular Investigation (SIDV-GIUV), Italy

Two very recent scientific papers ${ }^{1,2}$ have re-opened a debate on a vascular issue, chronic cerebrospinal venous insufficiency (CCSVI), that apparently was sent in a corner by other trials and some Editorial hasty conclusions. ${ }^{3,4}$ The never-ending debate is still open and, perhaps, a one-year truce helped to calm waters and sort out, as by means of a sandbox, the situation from the vascular point of view. Before discussing why these recent papers have widened the path for CCSVI, some mind refreshing is mandatory, since the opinions are spread in all directions and a concise summary may help for those that are newcomers in this issue.

The definition of Chronic Venous Disease is present in every medicine textbook and always regarded a pathological vein condition for the lower limbs. Hemorheological remedies, both medical and surgical, have so far principally aimed to relieve symptoms and correct hemodynamic aspects, improving quality of life and slowing a process that apparently cannot be stopped. The term chronic itself means that the disease cannot be completely removed and this condition should always be remembered.

Ten years ago, Paolo Zamboni opened our fossilized gaze from the lower limbs to the brain and reminded us that there is a venous return system in this district too. Like for the lower limbs, a cerebral venous return misfunction can give consequences and, coincidentally, this situation is most present in some progressive neurological diseases. At that time the attention was focalized on one specific category of disease: multiple sclero$\operatorname{sis}(\mathrm{MS}){ }^{5}$

Since then the Scientific World divided itself in believers, non-believers or sceptic and absolute negativists but the issue was on and, worldwide, thousands of Patients were obviously interested. In ten years, chronic cerebrospinal venous insufficiency generated about 600 papers for PubMed and over 20.000 for Google Scholar, regarding neuro- logic progressive diseases, mainly MS and Meniere's Disease, but not only. Probably no other vascular issue did greater scientific production than CCSVI in the past years but apparently something went crooked, along with others that went straight. Sorting out and explaining, simply, is not easy but work, time and foresight shouldn't go waisted. Somehow Patients believed that the improvement of the venous cerebrovascular outflow would have cured their disease, however severe it could be. Parallelly, this also revealed that all traditional drug therapy carried out so far didn't succeed in this direction.

A shadowy aspect was that the diagnosis for CCSVI was left mainly to a Duplex exam, not easy to perform for both the physician and for the patient. The layout and interpretation of the results from the Duplex exam was complicated for most physicians, either neurologists, vascular surgeons or radiologists, closing the circle into a small group of specialists. The paradox was that Family Associations became experts, further more than most of their Family Doctors.

The Duplex exams however produced a considerable amount of imaging that, if compared to a normal subject, was incredibly proving towards a series of morphological and hemorheological abnormalities present in the jugular system, as well as the vertebral plexus. The venous cerebral drainage was more or less compromised in about $90 \%$ of MS population that described a sequence of recurrent symptoms. ${ }^{6}$

Being the Duplex exam necessarily performed in two different positions, lying down $\left(00^{\circ}\right)$ and sitting upright $\left(90^{\circ}\right)$, several abnormalities were mainly present in the second condition and this led to misunderstandings.

The correction or remedy to the venous malfunctions, stenosis or abnormal valve function, was principally found in vein angioplasty (vPTA), where materials had great development and interventional physicians applied their skills.

The good news was that a considerable number of patients that underwent to vPTA treatment had symptom benefits, in some occasions immediate and long-lasting, in other situations rather mild, still useful but evanescent in short time and in other situations they were unfortunately null.

A four-year review on a significant population allowed to determine symptom benefits in time and on the severity of the MS disease, where the relapse-remitting (RR) certainly had better and perduring results against the more complex and chronic secondary-progressive (SP) and primary-progressive (PP). ${ }^{7}$

Initially the conviction of some intervention physicians was to inflate the PTA balloons to their maximum capacity, some-
Correspondence: Pietro M. Bavera, Vascular Surgeon and Diagnostician for Solferino Vascular Lab, Milano; Member of the Italian Society for Angiology and Vascular Medicine (SIAPAV); Member of the Italian Society for Vascular Investigation (SIDV-GIUV), Italy.

E-mail: pietrombavera@gmail.com

Key words: Vein angioplasty; CCSVI; magnetic resonance imaging; multiple sclerosis; vein stenosis; venography; Ménière disease.

Received for publication: 22 April 2020.

Accepted for publication: 25 April 2020.

This work is licensed under a Creative Commons Attribution 4.0 License (by-nc 4.0).

C. Copyright: the Author(s), 2020

Licensee PAGEPress, Italy

Veins and Lymphatics 2020; 9:9053

doi:10.4081/vl.2020.9053

times even with the kissing balloon technique, stressing far too much the vein endothelium. Gradually with time, experience achievement and improved materials, these techniques preferred homogeneous and precision ballooning, where Duplex scanning, phlebography and MRI worked together. The imaging, both Duplex and above all phlebographic and MRI, led to the approval of CCSVI as a pathology. CCSVI received an official recognition as a disease within UIP Boston World International Congress $^{8}$ and even included in medicine textbook..$^{9,10}$

The pathology made its way inside other clinical situations such as Ménière disease $^{11,12}$ and sudden hearing loss ${ }^{13}$ with an infinity of significant imaging and Duplex hemodynamic exams.

To boost CCSVI, the most significant results were attended with the Brave Dreams (BD) Trial, ${ }^{14}$ supported by the Emilia Romagna Regional Agency of Health. Unfortunately, it was completed in less than half of the Italian Centers that had matched all requirements for the Trial. Brave Dreams was a multicenter, prospective, randomized, double-blind, sham-controlled trial to evaluate the clinical efficacy and safety of balloon angioplasty in the extracranial veins of patients with MS and CCSVI. It was necessarily completed after the five targeted years missing enrolment of 315 multiple sclerosis (MS) patients, out of the initially intended 430. The fallout of some Centers was unexpected, especially after initially approved endorsement.

The Brave Dreams Trial turned to be into an underpowered work and highlights were placed upon conclusions that certified 
venous PTA as a safe procedure but promptly labelled as ineffective with the omission that more than $50 \%$ of the treated population indeed had received benefits, without this being the principal aim of the Trial. Moreover, despite the dramatic reduction of the patients' sample, the findings on the secondary progressive subgroup were analyzed separately from the relapsing-remitting one, and reported in an appendix. ${ }^{14}$ Almost two years after the first BD results, we can comment further observations that come from two new published Studies.

The first one in the February 2020 issue of Journal of Endovascular Therapy, where Dr Zamboni and Colleagues report a reanalysis of all patients enrolled in the Brave Dreams, but this time also including secondary/progressive MS patients. ${ }^{1}$

This Study may conveniently be identified as Brave Dreams 2 (BD2). A terrific work was completed by Giaquinta ${ }^{15}$ to create what probably is the highest consecutive number of venogram measurements ever performed by a single physician. In effect it permitted a re-analysis of all the venograms that were performed during the BD study with further interpretation and lecture that demonstrated how vein angioplasty had a significant effect upon the diseases' activity, quantified by freedom imaging of new cerebral lesion development on MRI and/or decreased activity of existing lesions at 12 months in a population that included SP MS patients in addition to the RR group. An improved interpretation of the venograms concluded that the identification of venous outflow benefits was evident and that these also matched with MRI evidence of reduced cerebral lesions. Moreover, the paper clearly confirms that hypoplasia, external compression and long bulky and hypomobile valve flaps are recognizable with the Duplex, without necessarily always requiring phlebography. On these types of CCSVI the double-blind shows that the flow is not re-established and plaques are formed exactly as in the sham group.

De facto it is a milestone paper for MS, because it proves the equivalence between re-established drainage flow and the absence of formation of new plaques, which is the anatomy-pathological essence of the Disease. It is proven with the utmost evidence. ${ }^{14,16}$

The second paper from January 2020 Annals of Vascular Surgery issue ${ }^{2}$ regards a randomized wait list control Study and vPTA in patients with multiple sclerosis. The work is a tremendous high definition analysis carried out at the University of Pisa with involvement of several different Units that cross match their results. The paper is not of easy interpretation for All but never- theless an excellent guideline and roadmap regarding materials and methods within the CCSVI issue. Unfortunately, it is shaped from a low number of cases and on a short time-lapse follow up. Under a practical point of view the study of the lumbar plexus, that cannot be treated at the moment, increases the interpretation in the whole. It concludes that benefits are present for treated MS patients with CCSVI and showed significant improvements of some clinical-functional aspects, such as fatigue, pain, QoL both mental and physical, anxiety, depression, attention, and urinary urgency but achieving disability improvement appears unlikely, in such a short period. The paper itself is a good start point but may benefit of greater numbers (patients) and a longer time-lapse follow up. The work is positive for completing the CCSVI long and winding road-map.

Concluding, CCSVI is a topic with still work in progress but at present these two papers are milestones in the correct direction. More proofs will come again, perhaps less frequently than once with benefit for quality, but all will certainly be strong of ten-year long experience and follow ups.

\section{References}

1. Zamboni P, Galeotti R, Salvi F, Giaquinta A, Setacci C, Alborino S, et al Brave Dreams Research Group. Effects of Venous Angioplasty on Cerebral Lesions in Multiple Sclerosis: Expanded Analysis of the Brave Dreams Double-Blind, Sham-Controlled Randomized Trial. J Endovasc Ther. 2019 Nov 17:1526 602819890110. doi: $10.1177 / 152660$ 2819890110. [Epub ahead of print] Erratum in: J Endovasc Ther. 2020 Feb;27(1):NP1.

2. Napoli V, Berchiolli R, Carboncini MC, et al. Percutaneous venous angioplasty in patients with multiple sclerosis and chronic cerebrospinal venous insufficiency: a randomized wait list control study. Ann Vasc Surg 2020; 62: 275-286.

3. Zivadinov R, Weinstock-Guttman B. Extracranial venous angioplasty is ineffective to treat MS Nature Reviews. Neurology 5 Jan 2018.doi:10.1038/ nrneurol.2017.180

4. Traboulsee AL, Machan L, Girard JM, et al. Safety and efficacy of venoplasty in MS: A randomized, double-blind, shamcontrolled phase II trial. Neurology. 2018,30:e1660e1668.

5. Zamboni P, Galeotti R, Menegatti E, et al. A prospective open-label study of endovascular treatment of chronic cere- brospinal venous insufficiency. J Vasc Surg. 2009;50:13481358, e1341-1343.

6. Laupacis A, Lillie E, Dueck A, et al. Association between chronic cerebrospinal venous insufficiency and multiple sclerosis: a meta-analysis. CMAJ. 2011;183:E1203-1212.

7. Bavera P. May symptoms of chronic cerebrospinal venous insufficiency be improved by venous angioplasty? Veins and Lymphatics 2015; vol 4:5400

8. Lee BB, Baumgartner I, Berlien P, et al; International Union of Phlebology. Diagnosis and treatment of venous malformations. Consensus document of the International Union of Phlebology (IUP): updated 2013. Int Angiol. 2015;34:97-149.

9. Paolo Zamboni, Sergio Gianesini, and Erica Menegatti. Chronic cerebrospinal venous insufficiency, in diseases of the veins and lymphatics. Oxford Textbook of Vascular Surgery Edited by Matthew M.Thompson, Robert Fitridge, Jon Boyle et al. Oxford University Press Aug 2016

10. Zamboni P. The contribution of extracranial venous drainage to neuro-inflammation in multiple sclerosis. In: Minagar A, ed. Neuroinflammation. London: Elsevier; 2018:579-599.

11. Di Berardino F, Alpini DC, Bavera PM, et al. Chronic cerebrospinal venous insufficiency in Meniere disease. Phlebology. 2015 May;30(4):274-9.

12. Alpini, D., Bavera, P., Di Berardino, F., Barozzi, S., Cecconi, P., Farabola, M., Mattei, V., Mendozzi, L., Pugnetti, L., \& Cesarani, A. (2016). Bridging the gap between chronic cerebrospinal venous insufficiency and Ménière disease. Veins and Lymphatics, 5(2). https://doi.org/ 10.4081/vl.2016.5687

13. Alpini D, Bavera PM, Di Berardino F, et al. Bilateral sudden sensorineural hearing loss and chronic venous cerebrospinal insufficiency: a case report. Phlebology. 2013 Aug;28(5):231-3.

14. Zamboni P, Tesio L, Galimberti S, Massacesi L, Salvi F, D'Alessandro R, et al ; Brave Dreams Research Group. Efficacy and Safety of Extracranial Vein Angioplasty in Multiple Sclerosis: A Randomized Clinical Trial. JAMA Neurol. 2018 1;75(1):35-43

15. Giaquinta A, Beggs CB, Veroux M, et al. Factors influencing the hemodynamic response to balloon angioplasty in the treatment of outflow anomalies of internal jugular veins. J Vasc Surg Venous Lymphat Disord. 2017;5(6):777-788.

16. Zamboni P, Zivadinov R. Extracranial Veins in Multiple Sclerosis: Is There a Role for Vascular Surgery? Eur J Vasc Endovasc Surg. 2018 Nov;56(5):618621. 